www.jmscr.igmpublication.org

Impact Factor (SJIF): 6.379

Index Copernicus Value: 71.58

ISSN (e)-2347-176x ISSN (p) 2455-0450

crossref DOI: https://dx.doi.org/10.18535/jmscr/v6i6.120

Journal Of Medical Science And Clinical Research

\title{
Clinical Pearl over Radiological Pitfall in Terminal Stage of Spondylolysis in Athlete: A Case Report
}

\author{
Ashay Jadhav', Anirban Mallick², Jahnavi Dande ${ }^{2}$, Simarpreet Singh Kalra ${ }^{1}$ \\ ${ }^{1}$ Postgraduate Resident, Department of Sports Medicine, NSNIS, Patiala, Punjab, India \\ ${ }^{2}$ Sports physician, Department of Sports Medicine, NSNIS, Patiala, Punjab, India
}

\begin{abstract}
Spondylolysis is an osseous defect of the pars interarticularis, thought to be a developmental or acquired stress fracture secondary to chronic low-grade trauma. It is encountered most frequently in adolescents, most commonly involving the lower lumbar spine, with particularly high prevalence among athletes involved in sports requiring repetitive hyperextension. Spondylolysis can be asymptomatic or can be a cause of spine instability, back pain, and radiculopathy. It is important to determine the staging and type of spondylolysis via radiological means in order to assess bony healing and return to play. Whereas MRI can be used for early detection, bone scan is used to differentiate between active lesion and chronic cases. Multislice CT with multiplanar reformats is the most accurate modality for detecting the bony defect and may also be used for assessment of osseous healing. The present report investigates into the radiological follow up and clinical recovery guiding return to play in an athlete with multi level terminal stage spondylolysis.

Keywords: spondylolysis, athlete, return to play.
\end{abstract}

\section{Introduction}

Spondylolysis is an acquired defect of the pars interarticularis which is either due to a growth disturbance or due to repetitive hyperlordotic trauma. It predicates spondylolisthesis and longterm complications including neural compression. Although genetic factors have been implicated, the major etiologic factor of spondylolysis remains repetitive stress associated with sport.

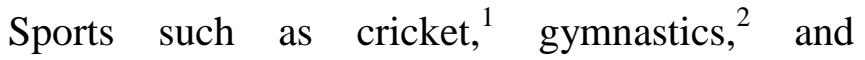
athletics, ${ }^{3}$ which particularly involve hyperextension and rotation of the lumbar vertebrae, expose the posterior elements of the immature spine to develop spondylolysis. ${ }^{4}$ It is a common cause of low back pain in children, adolescents, and young adults, with an incidence of $3-10 \%$ in the general population and $6 \%$ in adults. But only few reports of multiple level lumbar spondylolysis have been published. ${ }^{5}$ Multiple-level lumbar spondylolysis occurs more often in men than in women. ${ }^{6}$ In Athletic population, incidence ranges from $12 \%$ to $32 \% .^{7}$ Early detection of the defects will improve the outcome. Quantitative single-photon emission CT or MRI can provide important information for early detection or union of the pars defect, but progress of the union or morphological changes of the defect cannot be evaluated by these means. ${ }^{8}$ Unfortunately, neither MR nor CT and SPECT imaging can reliably distinguish whether an incomplete stress fracture is in the evolutionary or reparative phase. The following case report is an effort to establish the importance of clinical judgement in management of athlete and return to play (RTP) in the absence of radiological support. 


\section{Case Report}

A 22-year-old female elite heptathlete (height$166 \mathrm{~cm}$, weight- $63 \mathrm{~kg}$, BMI- $19.81 \mathrm{~kg} / \mathrm{m}^{2}$ ) presented with worsening low back pain (LBP) since 7 days. The complaint began approximately one month prior to presentation with insidious onset, dull aching in nature, more so on the left side. The pain was sharp during training particularly while doing high jump activity with a considerable hyperextension. The pain was not so during running activity and relieved by rest. Pain was non-radiating and not aggravated by coughing or sneezing. There was no past history of LBP and she has been training heptathlon for last 12 years. Her menstrual and dietary history were normal and family history was unremarkable. On examination para spinal muscle spasm is felt but no palpable step deformity. Lumbar flexion and extension both were limited.

Pain was aggravated during extension more so while standing on single leg and torso rotation. However, there were no neurological signs and symptoms. Knee and ankle reflexes were normal. There was no muscle weakness in quadriceps, hamstrings or calf muscles. X-ray LS spine AP, lateral and oblique views were advised, which showed mild disc space narrowing between L5 and S1. But, on oblique view X-ray (Figure 1) there was typical appearance of pars defect on L3 and L5.

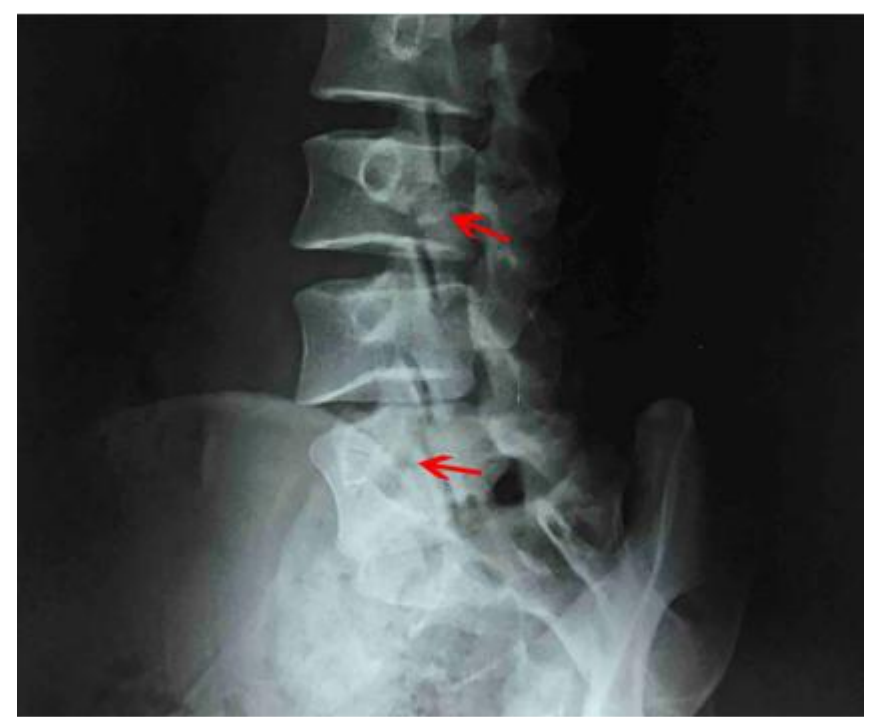

Figure 1 X-ray LS spine Oblique view showing typical appearance of pars defect on L3 and L5.

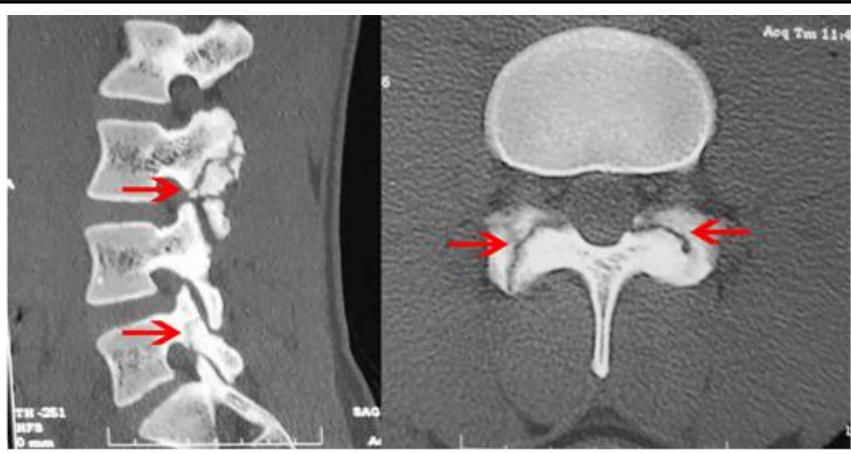

Figure 2- CT scan LS spine at the time of presentation.

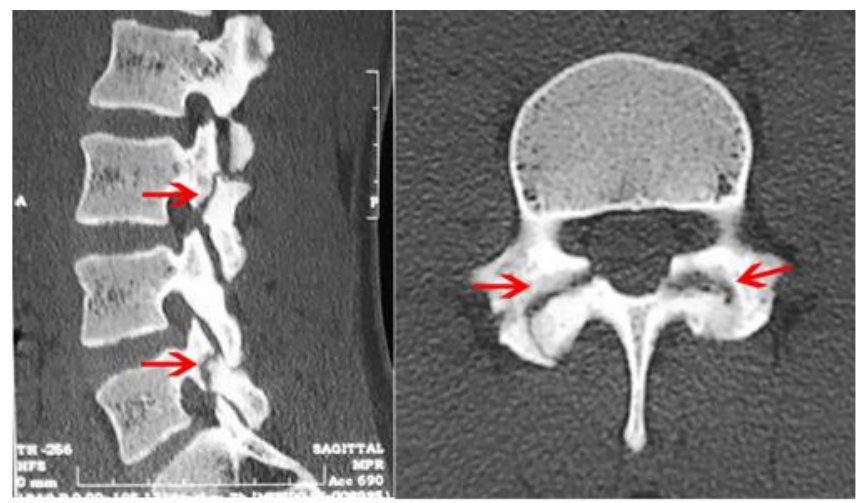

Figure 3- CT scan LS spine at RTP after 6 months of injury.

Hence, CT scan (Figure 2) was done taking 3x3 $\mathrm{mm}$ contiguous axial sections with sagittal and coronal reconstruction and it showed old fracture bilateral at L3 and L5 pars without significant displacement. Few small fracture fragments are also seen at fracture site. Slight sclerosis and irregularity of fracture are also seen at both levels.L1, L2 and L4 vertebra showed normal morphology and there was no evidence of spondylolisthesis at any level. The CT scan finding was consistent with terminal stage spondylolysis. Hence, the athlete was counselled regarding the poor chance of bone healing and she was advised not to involve in any sporting activity till the pain subsides. Brace was not given and she was advised a progressive core stabilization programme initially in neutral position then gradually in flexion protocol. Passive stretching of the lumbar and psoas muscles were advised. Once the pain subsided, rehabilitation was aimed for strengthening of the rectus abdominis, erector spinae and gluteus/hamstrings. 
After 4 months of rehabilitation, a repeat CT was done which showed no additional changes compared to the earlier one which actually confirmed the staging of our diagnosis. Athlete's pain was reduced considerably and she was advised to continue core stabilization programme and functional training. After another 2 months, CT (Figure 3) was repeated once again but still there were no signs of healing. By this time, the athlete was absolutely pain free even doing the provocative manoeuvre.

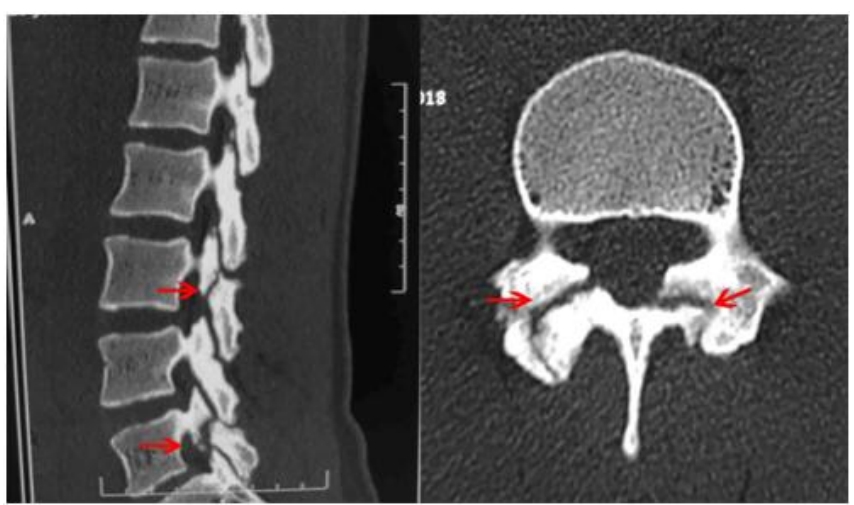

Figure 4- CT scan LS spine after 18 months of injury.

She was cleared to participate in training with a graduated return to play protocol. There was no recurrence of the same complaint for a follow up period of 12 months even though the CT scan was not showing any sign of bony healing. The athlete scored her career best in an international competition 18 months after the injury.

\section{Discussion}

CT, which provides excellent bony detail, is widely regarded as the reference standard for demonstrating complete and incomplete pars defects and for assessment of bony healing in the follow up of acute lesions. CT however, cannot reliably distinguish between active and inactive lesions and exposes the patient to ionizing radiation. SPECT is an extremely sensitive technique for early diagnoses of spondylolysis and may be predictive of the ability for lesion to heal. Unfortunately, SPECT is non-specific and cannot distinguish between stress reaction and overt fractures. That is why MR imaging, which has the advantage of not using ionizing radiation and has the potential to detect the bone marrow edema of the early stress reaction in the pars with the appropriate imaging sequences, should be used routinely as the first-line imaging modality for investigation of spondylolysis. Furthermore, an established lesion and other significant pathology (e.g., disc herniation) are also well demonstrated on MR imaging. It is also noted that a significantly greater proportion of defects achieved union in the early stage compared with progressive and terminal stages, and no bony union was seen in the terminal stage. ${ }^{9}$ Studies that compared conservative management strategies and bony healing did that based on radiological methods but clinical outcome was not reported for these studies. ${ }^{10}$ Hence it is important to evaluate and follow up these terminal stage injuries clinically. The current case is a perfect example for that but the patient needs to be followed up for recurrence and development of spondylolisthesis.

\section{References}

1. Hardcastle P H. Repair of spondylolysis in young fast bowlers. J Bone Joint Surg Br. 1993;75(3):398-402.

2. Vrable A, Sherman A L. Elite male adolescent gymnast who achieved union of a persistent bilateral pars defect. Am J Phys Med Rehabil. 2009;88(2):156-160.

3. Reitman C A, Esses S I. Direct repair of spondylolytic defects in young competitive athletes. Spine J. 2002;2(2):142144.

4. Iwamoto J, Sato Y, Takeda T, Matsumoto H. Return to sports activity by athletes after treatment of spondylolysis. World J Orthod. 2010;1(1):26-30.

5. Chung CH, Chiu HM, Wang SJ, Hsu SY, Wei YS: Direct repair of multiple levels lumbar spondylolysis by pedicle screw laminar hook and bone grafting: clinical, CT, and MRI assessed study. J Spinal Disord Tech 20:399-402, 2007 
6. Ogawa $\mathrm{H}$, Nishimoto $\mathrm{H}$, Hosoe $\mathrm{H}$, Suzuki N, Kanamori Y, Shimizu K:

Clinical outcome after segmental wire fixation and bone grafting for repair of the defects in multiple level lumbar spondylolysis. J Spinal Disord Tech 20:521-525, 2007.

7. Soler T, Calderon C. The prevalence of spondylolysis in the Spanish elite athlete. Am J Sports Med2000;28:57-62

8. K. Fujii, S. Katoh, K. Sairyo, T. Ikata, N. Yasui Union of defects in the pars interarticularis of the lumbar spine in children and adolescents the radiological outcome after conservative treatment. J Bone Joint Surg [Br] 2004;86-B:225-31.

9. L. Tonetti, A. Leone, M. Marino, A. Semprini, V. Zecchi, C.Colosimo; Lumbar spondylolysis: the role of imaging. Poster No.: C-0830, Congress: ECR 2016

10. Morita T, Ikata T, Katoh S, et al. Lumbar spondylolysis in children and adolescents. J Bone Joint Surg $[\mathrm{Br}]$ 1995;77: 620-5. 